\title{
Multidimensional scaling of form: A psychophysical analysis'
}

Both metric and nonmetric multidimensional scaling methods were used to analyze similarity estimates when random polygons were used as stimulus patterns. Three dimensions, dispersion, jaggedness, and elongation were obtained with both analyses and were related to physical measures of the patterns.

This research was designed to contribute to the development of a psychophysics of visual form perception and to the literature concerning how Ss make similarity judgments.

The psychophysical question was designed by Attneave and Arnoult over a decade ago (1956) and has been reviewed (Michels $\&$ Zusne, 1965) and re-evaluated more recently (Brown \& Owen, 1967). The basic problem is the determination of a multidimensional space for a given stimulus domain of forms which is defined independently of the perceptual behavior it is designed to predict (a physical space). Given a perceptually-relevant spatial representation of a stimulus domain, a multidimensional space may be constructed to represent some perceptual response (perceptual space) and the complete psychophysics would include the transformation relating these spaces. A psychophysics presumes a physics and no unique or generally accepted set of physical measures of visual form exists. Consequently, Brown and Owen (1967) have suggested an iterative procedure to develop such a measurement system. Essentially, they suggest the working hypothesis that some initial system represents the physical dimensions and dimensionality of the stimulus domain. They also suggest that multidimensional scaling techniques be used to define perceptual spaces, the structure of which may be compared to the physical space. Where lack of congruence obtains, revisions in the measurement system are suggested and continued iterations have the potential of developing a perceptually relevant physics. They have proposed such an initial measurement system and this study was designed in part to provide information concerning the degree to which it is relevant to perceived similarity.

One of the most critical problems which such a program encounters is the assumption that spatial models are appropriate tools for analyzing perceptual judgments. The technology of multidimensional scaling techniques has advanced much more rapidly than has knowledge concerning the appropriateness of spatial models to perceptual behavior. A number of specific problems have, however, received discussion.

The question of an appropriate metric has been most discussed. Attneave (1950) suggested a "city block" model for form stimuli, Torgerson (1952) has demonstrated the applicability of the Euclidian space to color judgments, and Shepard (1964) has studied the applicability of these two models to geometric forms. In a recent article, Hyman and Well (1967) reported a series of studies in which they used psychophysical procedures to compare alternative spatial models for the stimuli used by Attneave (1950), Torgerson (1952), and Shepard (1964). Their conclusions largely support previous work in that they conclude that the appropriate model depends upon the stimulus domain under study. In particular, the distinctiveness of component dimensions and the independence of dimensions both seem relevant. Were the problem to end here, it would be relatively simple since methods are available by which Minkowski r-spaces may be obtained (Kruskal, 1964a, 1964b) and the appropriate metric within this class becomes an empirical question for a given stimulus domain. However, it follows that difficulties arise with stimulus domains wherein differential distinctiveness exists for different dimensions of the same stimuli. Based upon present conclusions, it would not be expected that a single metric could be used for the entire space. That this may be true for some stimulus domains is suggested by the finding that some attributes of random polygons are more "accessible" for discrimination (Forsyth \& Brown, 1967) and that the same attributes become the focus of attention in a scaling task
(Forsyth \& Brown, 1968). Shepard (1964) has discussed the closely related problem that the appropriate metric may fluctuate with attentional processes.

In spite of these difficulties such procedures appear to be potentially very powerful tools for stimulus domains of unknown dimensionality and wherein an adequate psychophysics has been elusive. This study was designed as an initial evaluation of the use of existing techniques to help provide such data for a selected stimulus domain.

\section{Pat terns}

\section{METHOD}

Sixteen 4-sided random polygons were selected from a larger sample of 200 shapes which were generated at a constant area in a 100 by 100 unit matrix by a modification of Method 1 of Attneave and Arnoult (1956). The sample was selected on the basis of an analysis reported in detail elsewhere (Brown \& Owen, 1967) which attempted to determine interpretable, linearlyindependent, dimensions of this stimulus domain. Using 80 initial measures, factor analytic methods were used to reduce these to five factor-score measures which were interpreted as compactness, jaggedness, $y$-axis asymmetry, $x$-axis asymmetry, and rotation. The sample used herein was selected to be linearly independent with regard to these five attributes and to cover their range in the larger sample. The first of these goals was imperfectly realized due to the limited sample and linear correlations among factor scores ranged from .00 to .49. The shapes and their ordering on factor scores are shown in Fig. 1.

\section{Procedure}

Seventy-two undergraduates were presented with the 120 paired comparisons in a sequence derived by Ross (1934). Half of the Ss received pairs in the forward order and half received the backward order. They were instructed to rate pairs on the basis of their immediate impression of global similarity on a 7-point scale

\section{FACTOR}

\begin{tabular}{|c|c|c|c|c|c|c|c|c|}
\hline & 1 & 2 & & 3 & & 4 & & 8 \\
\hline & 1.11 & 1.01 & 1 & 1.76 & $r$ & 2.25 & 1 & 2.43 \\
\hline & .81 & .85 & 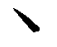 & 1.26 & $r$ & 1.40 & 1 & 1.41 \\
\hline & .78 & .85 & $\bullet$ & .79 & $\bullet$ & 1.20 & $>$ & .66 \\
\hline & .78 & .61 & $\bullet$ & .70 & - & 1.08 & 1 & .64 \\
\hline & .77 & .44 & - & .58 & $>$ & .84 & $r$ & .42 \\
\hline & .76 & .41 & $y$ & .48 & - & .47 & - & .32 \\
\hline & .74 & .29 & 1 & -.04 & $\bullet$ & .31 & $\checkmark$ & .15 \\
\hline & .54 & .10 & - & -.07 & $\bullet$ & -.22 & $\triangleright$ & .06 \\
\hline & .37 & .05 & - & -.16 & 1 & -.24 & $\nu$ & .05 \\
\hline & .18 & $-\quad .02$ & - & -.18 & - & -.48 & $\bullet$ & .04 \\
\hline & -.05 & $1-.36$ & $r$ & -.23 & 4 & -.58 & 4 & -.16 \\
\hline & -.42 & $1-.40$ & $r$ & -.53 & 1 & -.73 & $r$ & -.35 \\
\hline & -.61 & $>-1.68$ & $\checkmark$ & -.86 & $\checkmark$ & -81 & $y$ & -.60 \\
\hline & -.84 & -1.81 & $r$ & -.94 & 1 & -1.09 & $\bullet$ & -.79 \\
\hline & -1.77 & $/-2.11$ & $\checkmark$ & -1.07 & & -1.10 & - & -1.19 \\
\hline & 2.91 & $y-3.73$ & 7 & -1.82 & & -4.53 & - & -1.66 \\
\hline
\end{tabular}

Fig. 1. The ordering of shapes and their scores on the first five factors from an analysis of 80 shape measures. 


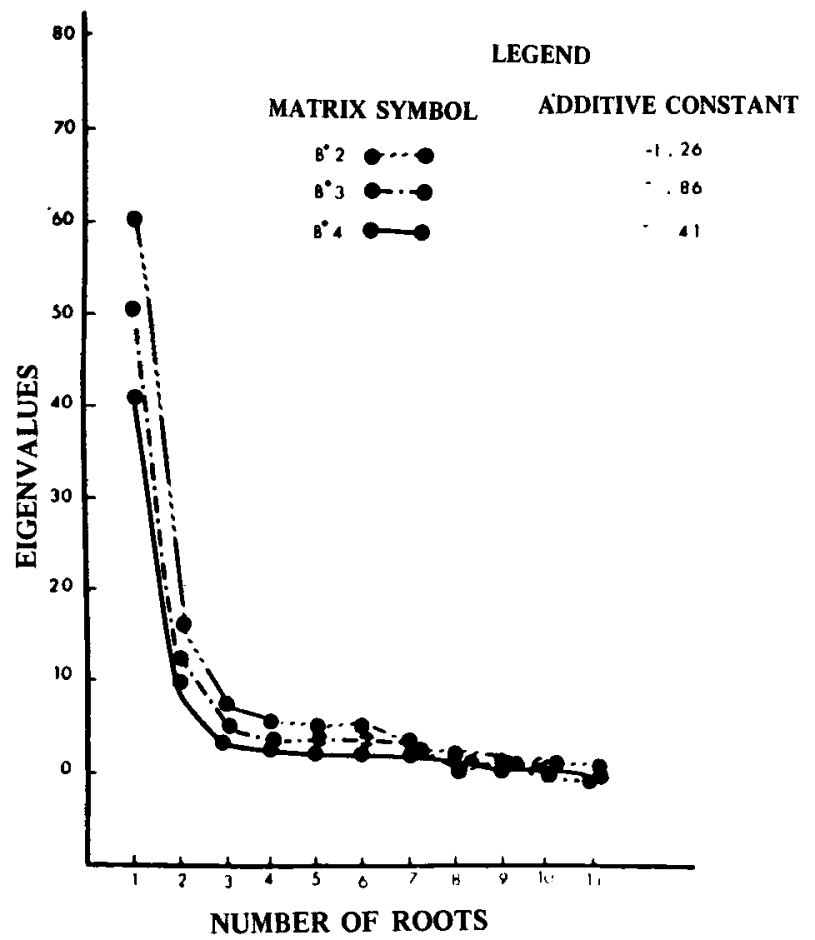

Fig. 2. The roots obtained with various numbers of factors from $B^{*}$ matrices of rank 2,3 , and 4.

ranging from extremely similar to extremely dissimilar. The scale was present throughout the task and scaling was preceded by 20 practice trials.

Pairs were presented in a dimly illuminated room by back projection on a viewing booth. Two tachistiscopically-shuttered random access projectors contained $35 \mathrm{~mm}$ slides of the shapes. At the beginning of each trial, $S$ depressed a plate causing the shutters to open and projecting a pair of patterns onto the viewing screen. When $S$ orally rated the pair and released the plate, the shutters closed and a red light was illuminated. When $E$ had selected the next pair, the light was extinguished as a signal to $S$ that the next trial could be initiated. This procedure was followed until all 120 pairs had been presented. Total testing time ranged from 40 to $60 \mathrm{~min}$.

\section{The Traditional Analysis}

\section{ANALYSES AND RESULTS}

The first of two analyses used what Torgerson (1965) has referred to as the "traditional approach" which requires that the perceptual space be Euclidian and that observed similarities among stimulus objects be linearly related to distances in that space. The multidimensional case of condition $\mathrm{D}$ of the law of categorical judgments (Torgerson, 1958) was used to reduce the 120 (shape-pairs) by 7 (categories) frequency matrix (F) to the 16 (shapes) by 16 (shapes) matrix $S=\left[s_{i i}\right]$ whose entries may be viewed as psychological distances among all patterns. A scalar products matrix $\mathrm{B}^{*}$ was computed placing the origin at the centroid of the points and the Messick and Abelson (1956) solution to the additive constant problem was applied to obtain absolute distances. The program which was utilized provides the option of obtaining a $\mathbf{B}^{*}$ matrix of any desired rank with a corresponding additive constant. Constants were obtained for matrices of ranks 2,3 , and 4 and each $B^{*}$ matrix was factored using a principal factor solution. Figure 2 clearly indicates a 3-dimensional solution, the three roots accounted for $90 \%$ of the variance in the selected $B^{*}$ matrix which is comparable to the results of other studies (e.g., Hyman \& Well, 1967), and the final 3-dimensional solution was highly interpretable. For these reasons, an orthogonal rotation of three factors was used to determine the final psychological dimensions and the projections of shapes on the factors as scale values.

The ordering of the shapes on the three dimensions with their projections on the orthogonal axes is shown in Fig. 3. The interpretation of these dimensions was referred to a psychophysical analysis.

The more elegant multivariate methods available for relating the two spaces were rejected in favor of a simple correlation and graphical analysis. Stenson (1960) has used orthogonal factor scores effectively as predictors. However, his factor scores were based upon an analysis of only the shapes used in his study. In the present case, orthogonality was sacrificed for generality. The factor scores were based upon a sample of 200 shapes and are, therefore, more representative of the stimulus domain. However, when subsets of shapes are sampled, the orthogonality may be lost (see Table 1). Instead, each dimension was correlated with the original 80 measures since the factor scores represent only the major linear dependence among the 80 measures and the advantage of orthogonality was lost. Selected relations were graphically plotted for visual inspection. Since these analyses obviously involve many correlations, and since the correlations among subsets of measures are very large, the 80 measures were reduced to a small set based upon "hypotheses" concerning which measures ought to predict performance. These measures were selected on the basis of their similarity to measures having previously-demonstrated predictive utility, and their relation to the factor scores. Where additional measures which were not selected on the basis of these criteria were uniquely predictive, the results are included.

The first physical factor was labelled compactness-dispersion (Brown \& Owen, 1967) and measures the extent to which the area or perimeter is dispersed away from the centroid of the shape. For fixed area, the length of perimeter $(P)$ provides an attractively simple measure. The second factor, jaggedness, refers to variability of angle sizes generally (at four sides it largely reflects the existence and size of an interior obtuse angle), and was represented by the variance of interior angles $\left(\mathrm{IA}_{2}\right)$. The third and fourth factors, skewness of area along the $y$ and $x$ axes respectively, were measured by the third moments of area units

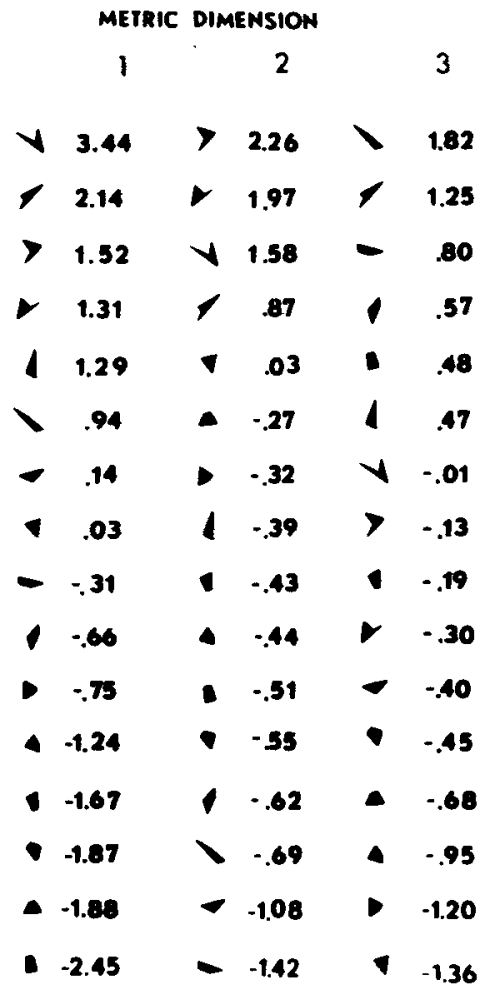

Fig. 3. Projections of shapes on the three orthogonal dimensions of the metric solution. 
Table 1

Correlations among Nonmetric Dimensions, Metric Dimensions, and Shape Measures (Decimels Omitted)

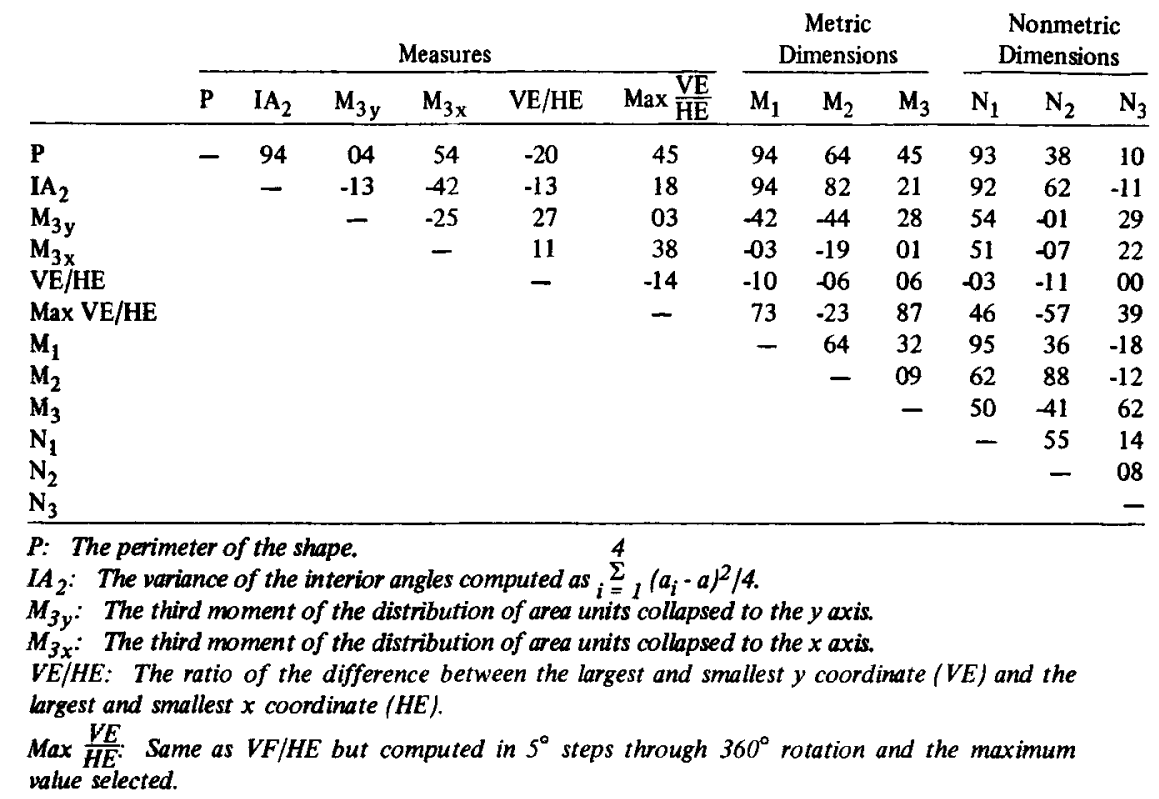

computed with regard to the appropriate axes. The last factor, rotation, was represented by the ratio between vertical and horizontal extent. The correlations (Pearson, product moment) among all independent and dependent variables are shown in Table 1.

The prediction and interpretation of the first two dimensions is complicated by a problem of dependence on both the independent and dependent variable sides. On the independent variable side, there is a very high linear correlation between the length of perimeter and the variance of interior angles (.94). This is

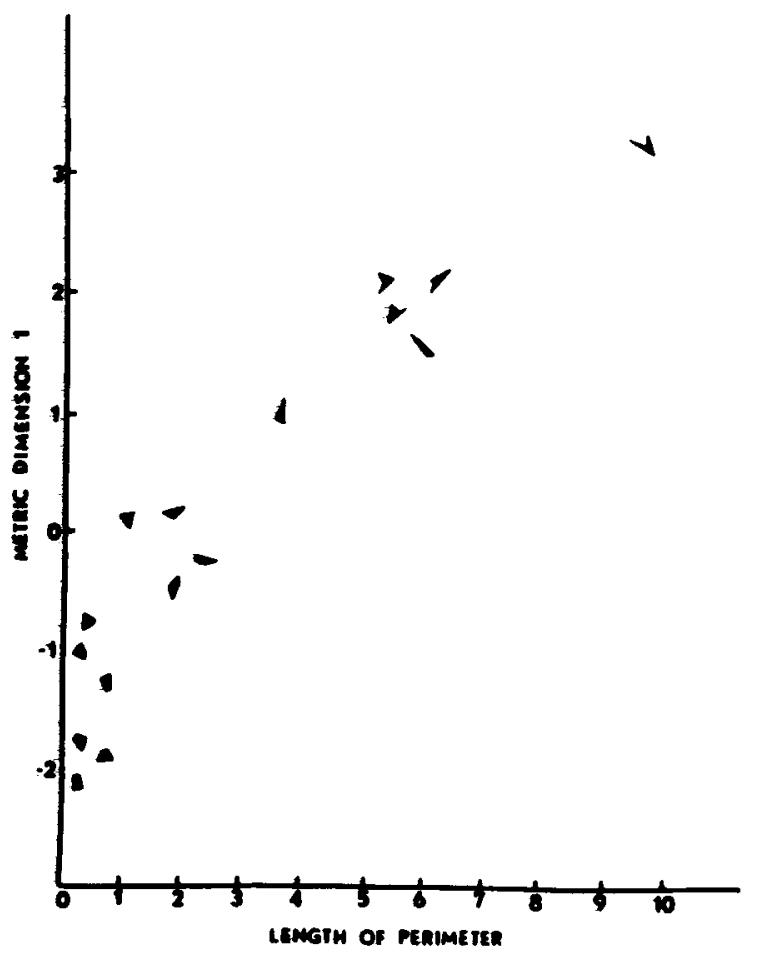

Fig. 4a. The plot of shape projections on dimension 1 against shape perimeter (rescaled to 1-10). perfectly logical with these shapes since a 4-sided form can have only one obtuse interior angle, and with fixed area, this increases the perimeter. Even though two orthogonal factors were obtained sampling over 200 shapes, this obviously did not guarantee orthogonality for these two measures over a limited sample. On the dependent variable side, the projections of the shapes on the first two perceptual dimensions are correlated to a moderate degree (.64). It is clear that the first dimension could be predicted equally well with either perimeter or the angle measure while the second dimension is best predicted from the angle measure. Some

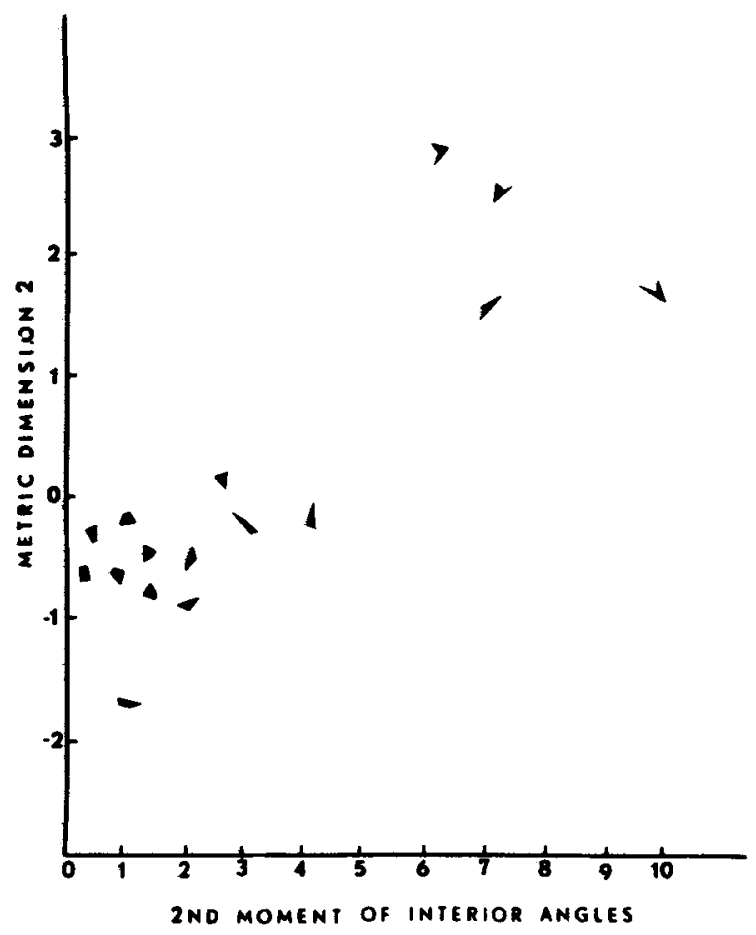

Fig. 4b. The plot of shape projections on dimension 2 against $\mathrm{IA}_{2}$ of the shapes (rescaled to 1-10). 


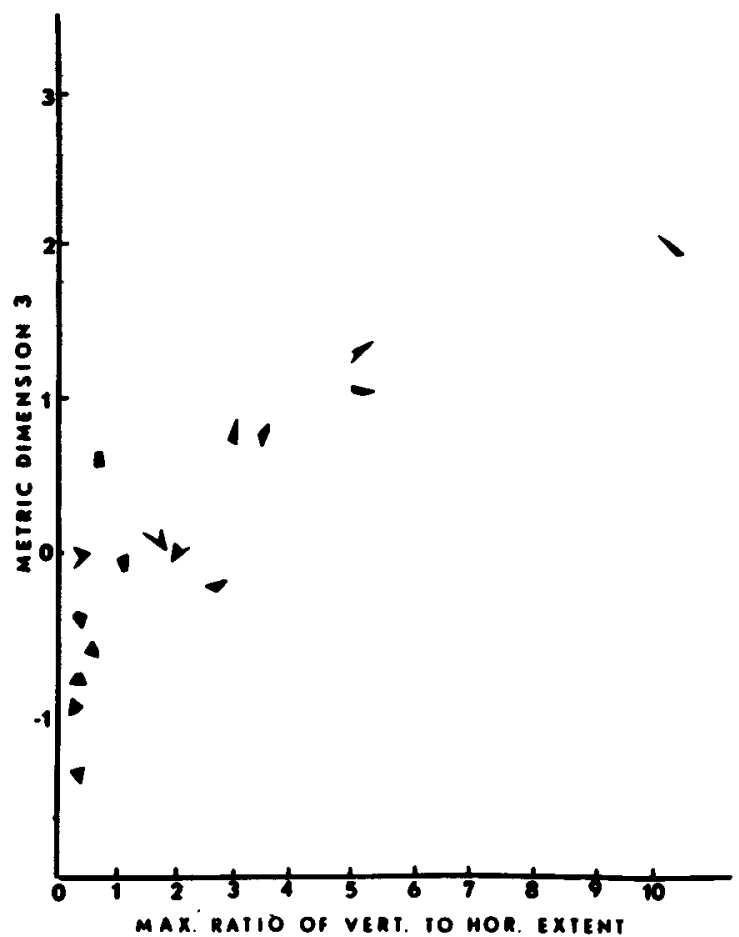

Fig. 4c. The plot of shape projections on dimension 3 against Max VE/HE (rescaled to 1-10).

interesting partial correlations were computed. Dimensions 1 and 2 were residualized with regard to their mutual linear dependence yielding the component in dimension 1 which is linearly independent of dimension $2\left(\mathrm{D}_{1} \cdot 2\right)$ and the residual in dimension 2 which is linearly independent of dimension $1\left(D_{2} \cdot 1\right)$. Similarly, perimeter and $I A_{2}$ were mutually residualized yielding $P \cdot I A_{2}$ and $I A_{2} \cdot P$. The partial correlations, $\left.r_{(P} \cdot I A_{2}\right)\left(D_{1} \cdot D_{2}\right)$ $\left.=.48, \mathrm{r}_{(\mathrm{P}} \cdot \mathrm{IA}_{2}\right)\left(\mathrm{D}_{2} \cdot \mathrm{D}_{1}\right)=.34, \mathrm{r}_{\left(\mathrm{IA}_{2}\right.} \cdot \mathrm{P}_{)}\left(\mathrm{D}_{1} \cdot \mathrm{D}_{2}\right)=.49$, and $\left.\mathrm{r}_{\left(1 \mathrm{~A}_{2}\right.} \cdot \mathrm{P}\right)\left(\mathrm{D}_{2} \cdot \mathrm{D}_{1}\right)=.33$ were computed. Obviously, these correlations do not help discriminate the best independent linear relations between $P$ and $I A_{2}$ and the two dimensions, since either measure independently predicts the residual variance in either dimension equally well. They make it clear that a large portion of the predictable variation in the dimensions is shared by the two measures. When the first two dimensions are plotted against $P$ (Fig. 4a) and $\mathrm{IA}_{2}$ (Fig. 4b), respectively, the nature of the correlations, however, becomes more clear. In the case of $D_{1}$ dispersed shapes which are not jagged receive large values on $D_{1}$ along with the jagged shapes, supporting the perimeter interpretation. In the case of $\mathrm{D}_{2}$, shapes were clustered into jagged and non-jagged shapes without regard to perimeter as such.

Dimension 3 was not highly correlated with either of the other two dimensions or with any of the other measures selected on an a priori basis. Its highest correlation was with the maximum ratio of vertical to horizontal extent. This measure is, essentially, a measure of the elongation of a shape without regard to any particular axis of orientation. The semi-partial correlations between Max VE/HE and $D_{3}$ with $P$ and $I A_{2}$ partialled out of Max $\mathrm{VE} / \mathrm{HE}$ were .75 and .84 , respectively. Dimension 3 is shown plotted against Max VE/HE in Fig. 4c.

An oblique rotation of three factors, utilizing a biquartimin criterion (Harman, 1960) resulted in a structure very similar to the orthogonal space. The correlations among the three primary axes were small $\left(r_{12}=.32, r_{13}=.05, r_{23}=.12\right)$ and the absolute value of the correlations over shapes between corresponding dimensions from the two solutions were dispersion, .99; jaggedness, .93; and elongation, .95 .
The Non-metric Analysis

The Kruskal (1964a, 1964b) nonmetric analysis was also applied to the data in order to compare the configuration based only upon rank order information with the results of the fully metric analysis and to evaluate the results with other than the Euclidian metric. The iterative procedure was used with Minkowski $r$-metrics of $r=1$ (city block model), $r=2$ (Euclidian model), and $r=3$.

Figure 5 shows the per cent stress plotted against the number of dimensions for each metric. While the stress curve fluctuates erratically "with the city block model, the other two curves drop smoothly with the sharpest break and lowest stress occurring with the Euclidian solution in three dimensions. Due to these data and the interpretability of the Euclidian results from the traditional analyses, the data presented are limited to that solution. Table 1 shows the linear correlations among measures, dimensions of the Kruskal solution, and dimensions of the metric solution. Figure 6 shows the plot of the nonmetric dimensions against the metric dimensions and the physical measure with which it was most highly correlated. The three dimensions each correspond most clearly to one of the metric dimensions and, in each case, has its highest correlation with the appropriate physical measure. It is worth noting that ordering of the shapes on the first and second nonmetric dimensions were correlated (.55), that both $\mathrm{P}$ and $\mathrm{IA}_{2}$ were highly correlated with the first dimension, and that no physical measure was highly correlated with the third nonmetric dimension. Of the 80 measures $P$ had the highest correlation with the first dimension. The second dimension correlated higher than .62 with only one measure (.72), the third moment of interior angles. This measures the skewness of angle sides and is correlated .96 with $\mathrm{IA}_{2}$. The third dimension was correlated greater than .39 with only one measure (.72), the variance of the second moments of area around the $x$ axis. This latter measure is rather complex. The second moment of area around the $x$ axis $\left(M_{2 x}\right)$ measures the dispersion of area relative to the horizontal axis. The shape was rotated in 5 deg steps, $\mathbf{M}_{2 \times}$ was computed at each orientation and the variance of these values was computed. This measures elongation or dispersion of area along any axis. It was highly correlated with Max VE/HE (.94), and it was correlated .80 with the elongation dimensions from the metric solution.

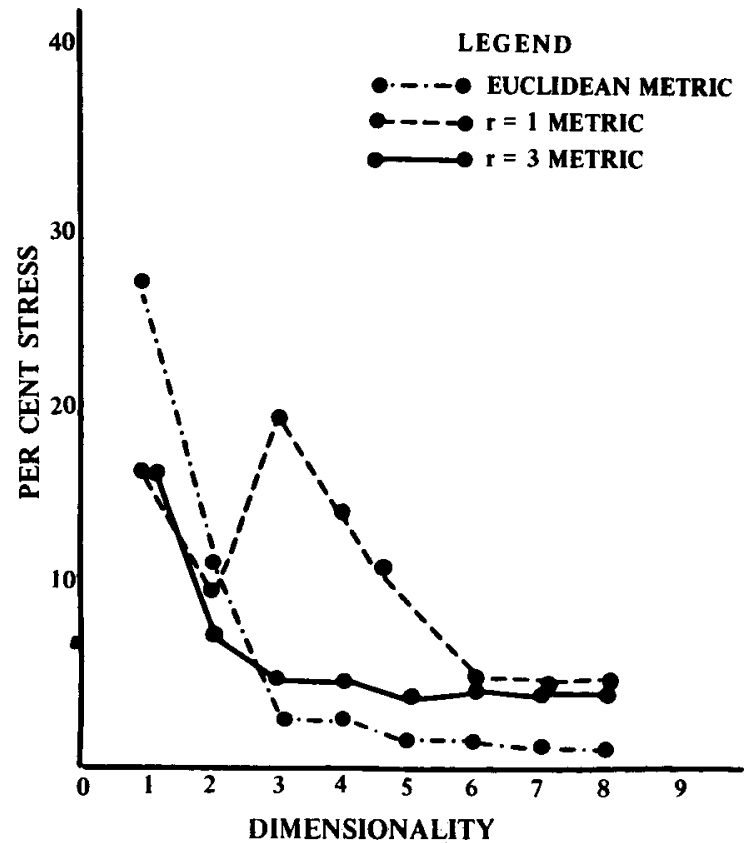

Fig. 5. Stress values for Minkowski r-metrics with varying number of dimensions. 


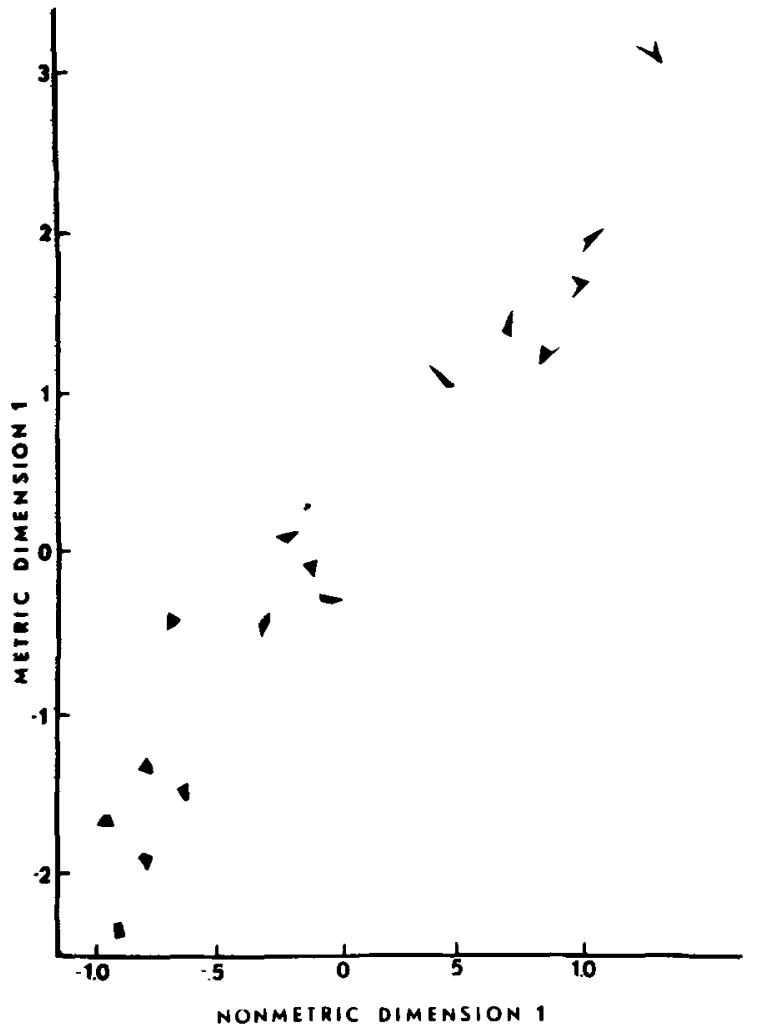

Fig. 6a. The plot of shape projections on the "compactness" dimension from the metric and nonmetric solutions.

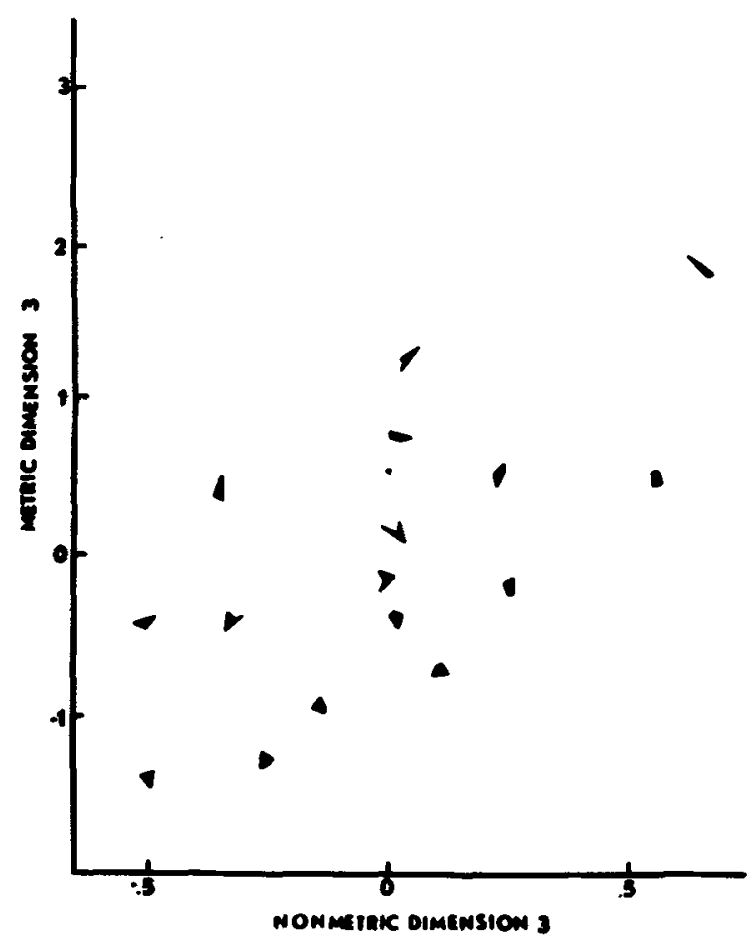

Fig. 6c. The plot of shape projections on the "elongation" dimension from the metric and nonmetric solutions.

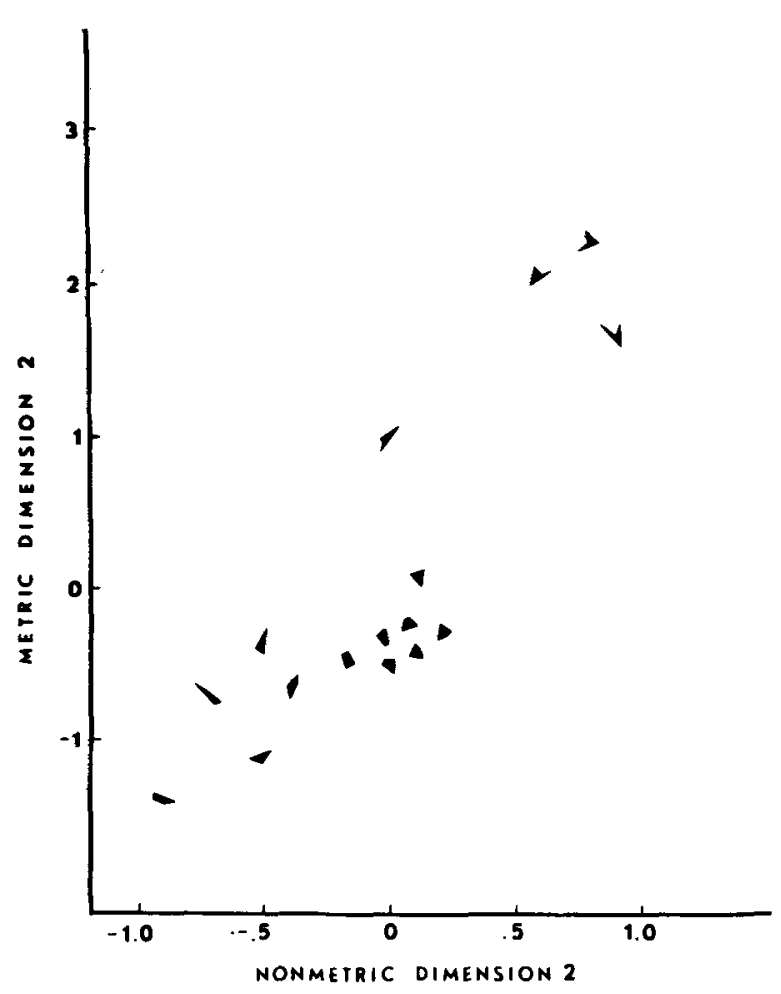

Fig. 6b. The plot of shape projections on the "jaggedness" dimension from the metric and nonmetric solutions.

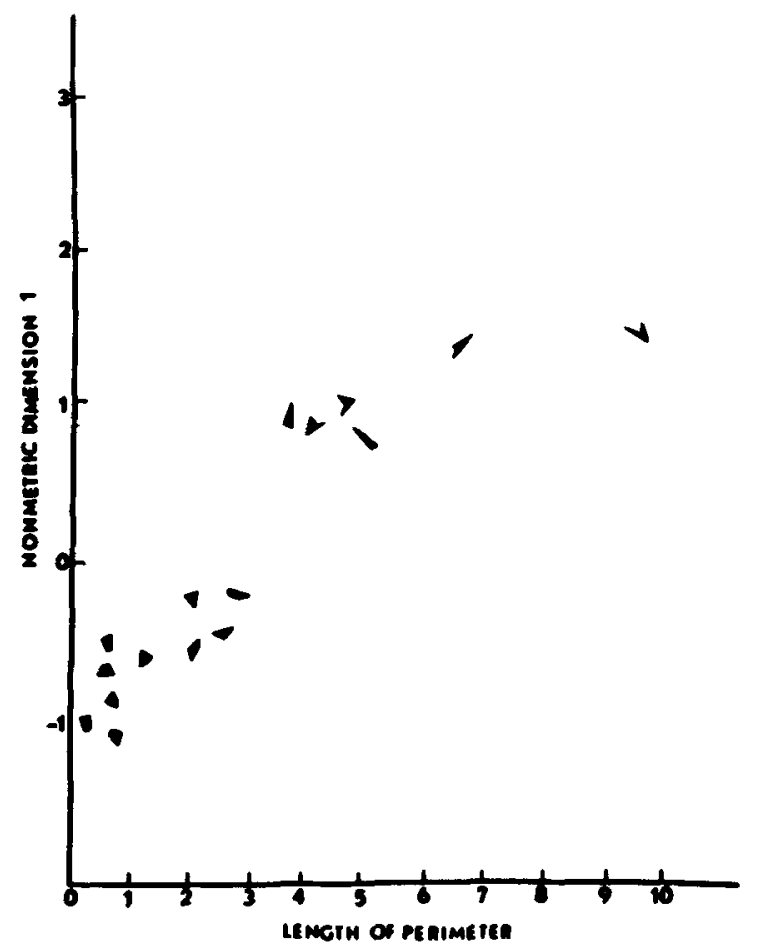

Fig. $6 d$. The relation between $P$ and projections of the shapes on the first nonmetric dimension. 


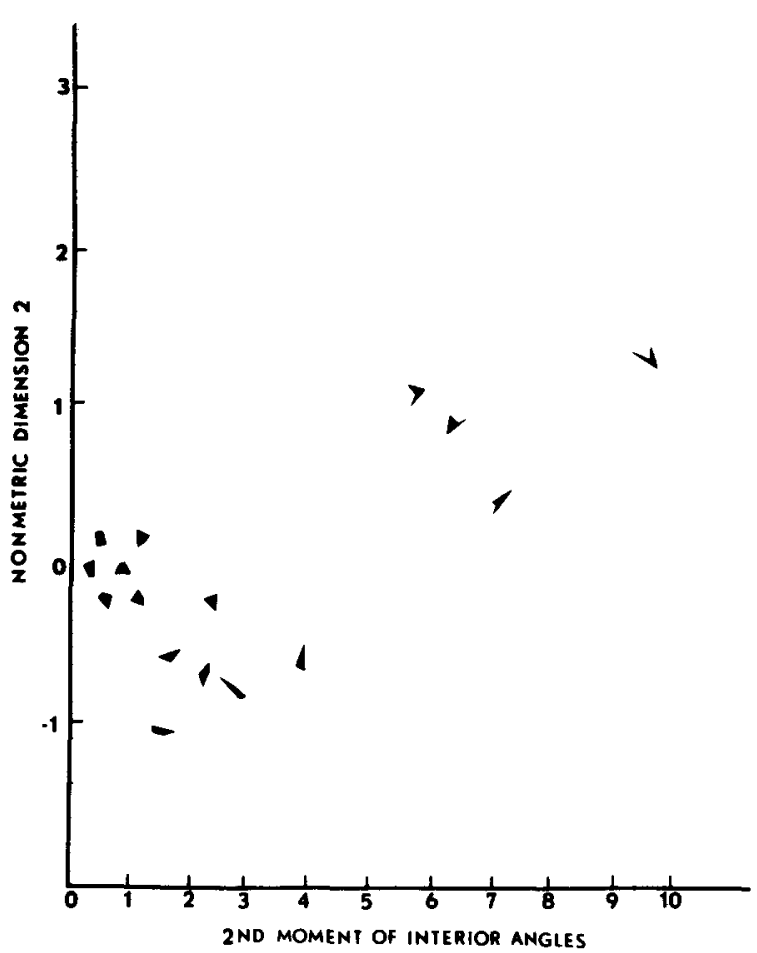

Fig. 6e. The relation between $I_{2}$ and projections of the shapes on the second nonmetric dimension.

\section{DISCUSSION}

Garner (1962) has suggested that speech, stimulation organized in time, is composed of, or may be represented in terms of, a multidimensional system of stimulus parameters. He and others (e.g., Attneave, 1959) have, in addition, discussed the importance of the logan-metron distinction when dealing with multivariate sources of stimulation. The view herein, and elsewhere (e.g., Brown \& Owen, 1967), has been that the visual perception of pattern, stimulation organized in space 2 , may be fruitfully studied when viewed in this same manner and that multidimensional scaling methods are well fitted to aid in such a program.

The results viewed from the point of the scaling models are encouraging. With forms, the dimensions of which are probably less distinctive than for those previously used, interpretable results were obtained. This is in spite of the fact that some correlation among these attributes was present in this sample. It is interesting that Ss were apparentiy sensitive to this correlation and it was, in part, reproduced in the responses. These data do not allow definitive statements to be made concerning the appropriate metric. The unusual stress results with the city block solution are probably due to a local minimum problem (Kruskal, 1964b) which is particularly acute at the extremes of these metrics. This could have been investigated by varying the starting configuration. However, due to interpretability, convenience, and good fit with the Euclidian results, this was not pursued. These results do support the general conclusion that the Euclidian model provides an adequate initial model for this stimulus domain. This is in contrast to the general conclusion of others (Attneave, 1950; Hyman \& Well, 1967; Shepard, 1964) who favor a city block model with their patterns. This is a difficult question and more careful studies are required before sound conclusions will be reached.

The psychophysical results support previous results in which the predictive utility of measures similar to these has been demonstrated. Attneave (1957), Arnoult (1960), and Owen \& Brown (1966) have demonstrated that dispersion measures $\left(\mathrm{P}^{2} / \mathrm{arca}\right.$, or $\mathrm{P}$ with area invariant), and angular variability incasures predict unidimensional judged complexity when independent sides is

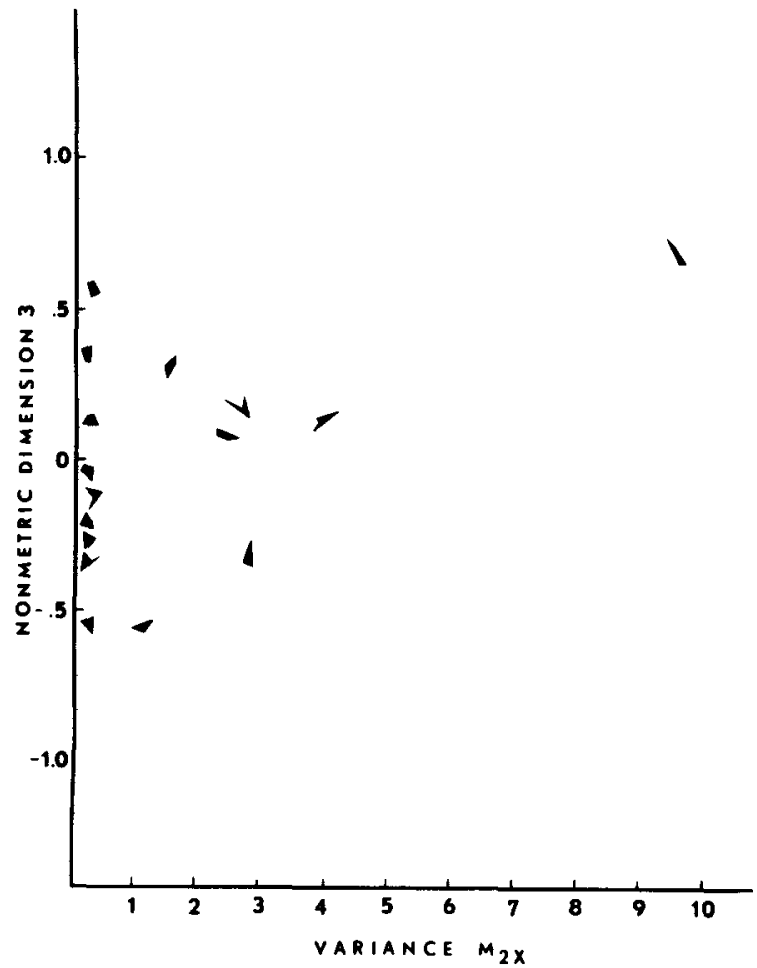

Fig. 6f. The relation between the variance of $M_{2 x}$ and projections of the shapes on the third nonmetric dimension.

already a predictor. Stenson's (1966) major physical factor was highly correlated with $\mathbf{P}, \mathbf{P}^{2} /$ area, and angular variability, and was correlated .90 with unidimensional complexity judgments. Finally, in the only multidimensional study on complexity (Silver, Landis, \& Messick, 1966), these same measures predicted average dimensions as well as dimensions for individual $S$ points of view. It seems clear that multidimensional scaling results are meaningful with such domains and can be predicted from a relatively small set of shape measures. The possibility of using the subjective dimensions to improve the definition of the physics, however, requires further work. It is obvious that the dimensions were not simply linear functions of the measures. The jaggedness results were essentially bipolar for these data; the perimeter-dispersion relation was monotonic, but not linear. The obvious requirement is to study the behavior of these dimensions with respect to the classical unidimensional scaling questions. These data help reveal the logan content of the domain, but the question of discriminable units and their relation to physical measures remains to be investigated. Indeed, the whole question of the behavior of individual dimensions extracted from multidimensional results is largely uninvestigated in terms of the traditional questions of unidimensional psychophysics or the more recent questions of uncertainty analysis.

\section{REFERENCES}

ARNOULT, M. D. Prediction of perceptual responses from structural characteristics of the stimulus. Percept. mot. Skills, 1960, 11, 261-268.

ATtNeave, F. Dimensions of similarity. Amer. J. Psychol., 1950, 63, 516-556.

ATTNEAVE, F. Physical determinants of the judged complexity of shapes. J. exp. Psychol., 1957, 53, 221-227.

ATTNEAVE, F. Applications of information theory to psychology. New York: Holt, Rinehart, \& Winston, 1959.

ATTNEAVE, F., \& ARNOULT, M. D. The quantitative study of shape and pattern perception. Psychol. Bull., 1956, 53, 452-471.

BROWN, D. R.. \& OWEN, D. H. The metrics of visual form: methodological dyspepsia. Psychol. Bull., 1967, 68, 243-259. 
FORSYTH, G. A., \& BROWN, D. R. Stimulus correlates of tachistiscopic discrimination-recognition performance: compactness, jaggedness, and areal asymmetry. Percept. \& Psychophys, 1967, 2, 597-600.

FORSYTH, G. A., \& BROWN, D. R. Stimulus recognizability judgments as a function of the utility of physical dimensions in recognition-discrimination problems. Percept. \& Psychophys., in press.

GARNER, W. R. Uncertainty and structure as psychological concepts. New York: Wiley, 1962.

HARMAN, H. H. Modern factor analysis. Chicago: Univ. of Chicago Press, 1964.

HYMAN, R., \& WELL, A. Judgments of similarity and spatial models. Percept. \& Psychophys, 1967, 2, 233-248.

KRUSKAL, J. B. Multidimensional scaling by optimizing goodness of fit to a nonmetric hypothesis. Psychometrika, 1964a, 29, 1-27.

KRUSKAL, J. B. Nonmetric multidimensional scaling: a numerical method. Psychometrika, 1964b, 29, 28-42.

MESSICK, S. J., \& ABELSON, R. P. The additive constant problem in multidimensional scaling. Psychometrika, 1956, 21, 1-15.

MICHELS, K. M., \& ZUSNE, L. Metrics of visual form. Psychol Bull, 1965, $63,74-86$

OWEN, D. H., \& BROWN, D. R. Physical correlates of pattern perception for the visual and tactual modalities. Paper presented at the American Psychological Association, New York, 1966.
ROSS, R. T. Optimum orders for the presentation of pairs in the method of paired comparison. J. educ. Psychol, 1938, 25, 375-382.

SHEPARD, R. N. Attention and the metric structure of the stimulus space. $J$. math. Psychol, 1964, 1, 54-87.

SILVER, C. A., LANDIS, D., \& MESSICK, S. Multidimensional analysis of visual form: an analysis of individual differences. Amer. J. Psychol, 1966, 79, 62-72.

STENSON, H. H. The physical factor structure of random forms and their judged complexity. Percept. \& Psychophys., 1966, 1, 303-310.

TORGERSON, W. S. Multidimensional scaling: I. Theory and method. Psychometrika, 1952, 17, 401-419.

TORGERSON, W. S. Theory and methods of saling. New York: Wiley, 1958.

TORGERSON, W. S. Multidimensional scaling of similarity. Psychometrika, $1965,30,379-393$.

\section{NOTES}

1. This research was supported by Research Grant HD-00909 from the National Institute of Child Health and Human Development.

2. This is not to deemphasize the fact that the visual perception of form may involve temporal organization.

(Accepted for publication March 20, 1968.) 